المجلة الدولية للدراسات الإسلامية المتخصصة

بحث رقم 2

المجلد الخامس، العدد الثانبي، 78:65 كانون الأول (2020)

\title{
الليبرالية الإسلامية في سنغافورة دراسة تطبيقية واقعية
}

محمد حفيظ الدين بن خميس

طالب بكالوريوس- قسم أصول الدين

جامعة اليرموك- الأردن
رائد سعيد بنـي عبد الرحمن

أستاذ مساعد في العقيدة- كلية الشريعة

جامعة اليرموك- الأردن

raed.said@yu.edu.jo 


\title{
الليبرالية الإسلامية في سنغافورة- دراسـة تطبيقية و اقعية
}

\author{
رائد سعيد بني عبد الرحمن \\ أستاذ مساعد في العقيدة- كلية الشريعة- جامعة اليرموك- الأردن \\ raed.said@yu.edu.jo \\ متمد حفيظ الدين بن خميس \\ طالب بكالوريوس- قسم أصول الدين- جامعة اليرموك- الأردن
}

DOI: https://doi.org/10.31559/SIS2020.5.2.2

الملخخ:

يهدف البحث إلى بيان مفهوم الليبرالية الإسلامية عند المجتمع السنغافوري، من خلال الكشف عن حقيقتها والأسباب التي

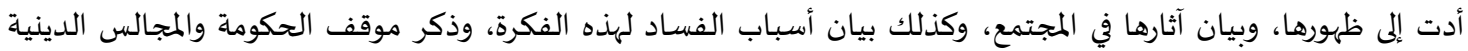
والفتاوى منها. وتتجه الدراسة نحو الاعتماد على المنهج الوثائقي المقارن، والمنهج التحليلي.

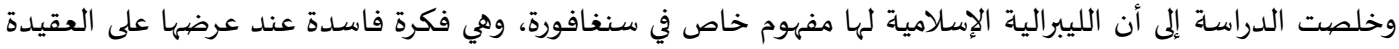

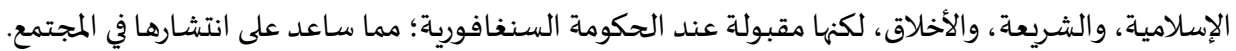

الكلمات المفتاحية: المذاهب؛ الفكرية؛ المعاصرة؛ الليبرالية الإسلامية؛ سنغافورة؛ الحرية؛ المجلس الديني الإسلامي؛ حقوق

الإنسان؛ العلمانية.

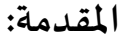

الحمد لله الذي خلق الإنسان في أحسن تقويم، وجعلنا من المسلمين, وأمرنا بالتقوى والخلق الكريم، وأشهد أن لا إله إلا الله وحده لا اللا

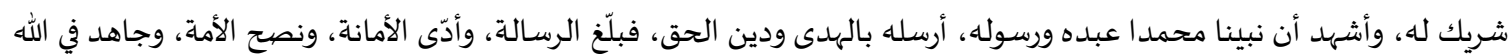

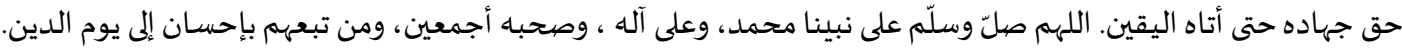

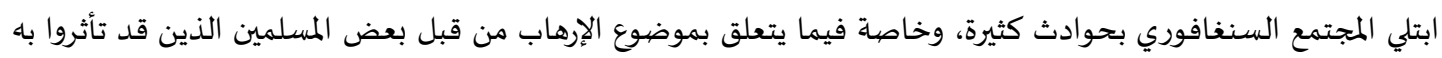

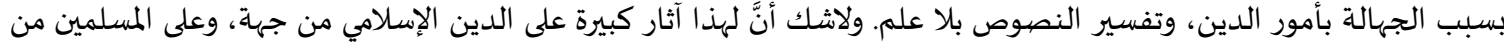

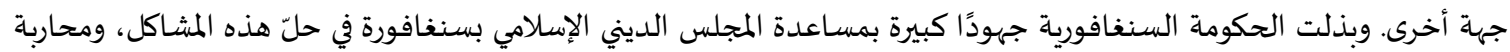
هذه التهديدات، ومراعاة الأمن، والسلامة داخل البلد.

وتحت هذه الشعارات، ساعدت الحكومة على غزو الفكر، وإنبات التشكيك والتشويش بالدين الإسلامي، وإيمان الفرد المسلم.

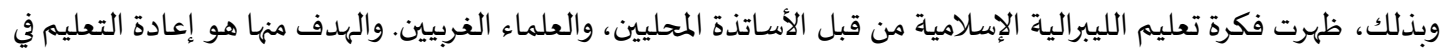

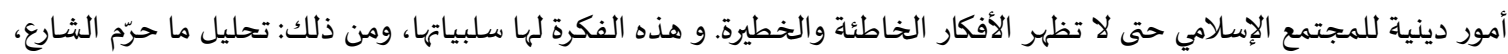

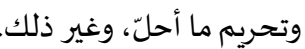
ولهذا السبب، وقع الاختيار على فكرة البحث؛ لضرورة كشف حقيقة هذه الفكرة، والمفسدة المترتبة عليها، وذلك لأنها تتعلق بعقيدة الفرد ونجاته في الدارين. 
مشكلة الدراسة وأسئلتها:

إن المتأمل في المجتمع السنغافوري يرى كثرة الأفكار، والآراء الغريبة الواقعية ظاهرها وباطنها، وهذه الأفكار تعارض النصوص لنها الشرعية، ، لكها سليمة ومقبولة في عيون الحكومة خاصة،، والمجتمع السنغافوري عامة. والأكبر من ذلك أنها تتفق مع القوانين والقيم

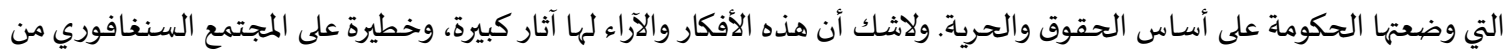

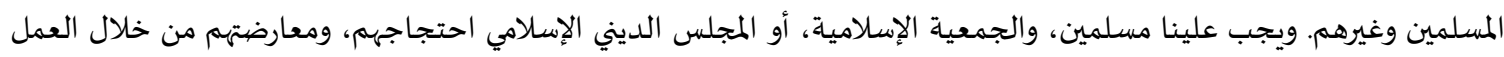

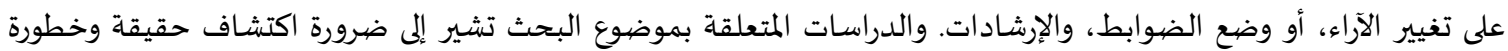
الليبرالية الإسلامية.

وتحاول الدراسة الحالية الإجابة عن الاسئلة الآتية:

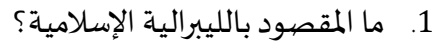

2.

3. ما آثار هذه الفكرة على المجتمع السنغافوري؟

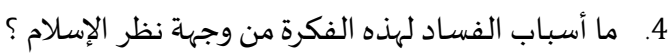

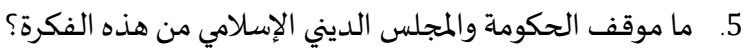

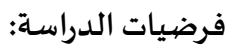

تتمثل فرضيات الدراسـة فيما يأتي:

1. ت تأثر المجتمع المسلم بهذه الفكرة.

2. انتشار فكرة الليبرالية الإسلامية بشكل واسع في المجتمع السنغافوري.

3. سمحت الحكومة بهذه الفكرة في المجتمع السنغافوري.

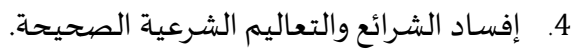

5. النزاع الدائم بين المسلمين في المجتمع.

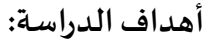

تتمثل أهداف الدراسة الحالية في:

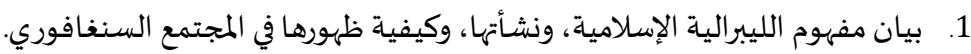

2. الكشف عن آثار هذه الفكرة في المجتمع السنغافوري.

3. بيان موقف الحكومة والمجلس الديني من هذه الفكرة.

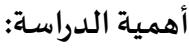

1. انتشار الفكرة بشكل واسع على أرض الواقع في سنغافورة.

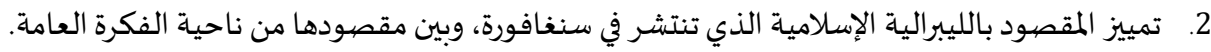

3. تطبيق الفكرة حتى تظهر الأفكار والآراء الغرببة في المجتمع السنغافوريك.

4. ضرورة معرفة خطورة هذه الفكرة على المسلمين خاصةة، وغيرهم عامة.

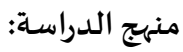

يعتمد البحث على المنهج الوثائقي، والمنهج التحليلي، وذلك من خلال الخطوات الآتية:

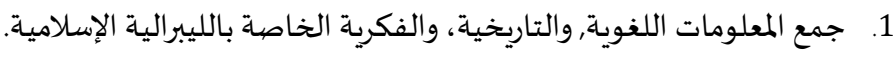

2. جمع أقوال الحكومة، وأقوال المجلس الديني الإسلامي عن هذه الفكرة.

3. ذكر الطرق التي تسلكها الحكومة في تطبيق الفكرة على المجتمع السنغافوري وبيان آثارها.

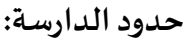

تقتصر الدراسة الحالية على النحو الآتي: 


$$
\text { 1. }
$$

مصطلحات الدراسة:

تتمثل مصطلحات الدارسة بما يلي:

• الليبرالية الإسلامية: المحاولة في إعادة تفسير الدين والنصوص حسب تجدئ لتدد الزمان، والواقع حتى يبقى الدين مناسبًا وصوابًا ومعتدلًا ضمن الجيل الحديث؛ ولمحاربة الأيديولوجيات المتطرفة.

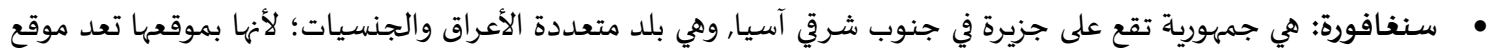

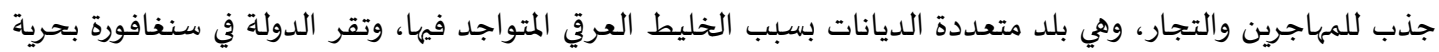
الاعتقاد، وفصل الدين عن الدولة. • دراسة تطبيقية و اقعية: هي دراسة علمية تعبر عن القضايا الراهنة، والحقيقية العملية مما لها علاقة وأثر على الحياة الفردية، والاجتماعية في بلد معين.

الدارسات السابقة:

كان من أبرز الدارسات ذات الصلة بموضوع الدارسة ما يأتي:

هراسة أحمد علي نور الدين (2005) بعنوان: ISLAM AND STATE: A STUDY OF THE LIBERAL ISLAMIC NETWORK

IN INDONESIA, 1999-2004

هدفت الدراسة إلى بيان شأن حركة الليبرالية الإسلامية في إندونيسيا من سنة 1999م إلى 2004م. وقد خلصت الدراسة إلى أن

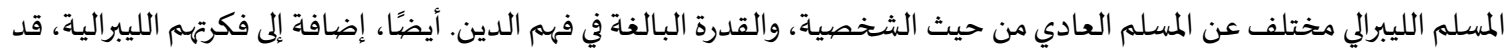
تؤدي مراراً إلى ظهور الآراء والأفكار المختلفة نحو المسألة المعينة عن غيرهم. وسلك الباحث في الدراسة المنهج الوثائقي والتحليلي. وتتفق دراسة أحمد علي نور الدين مع الدراسة الحالية في بيان سبب ظهور هذه الفكرة، ومن جانب آخر إثبات وإنبات وجود الآثار الخطيرة

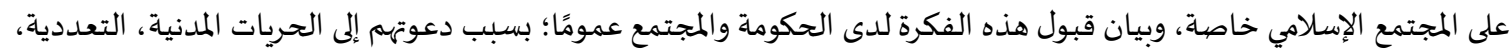
مساومة المرأة، فتح باب الاجتهاد، وغيرها. وتتميز الدراسة الحالية عن دراسـة أحمد علي نور الدين في بيان الطرق التي تسلكها الحكومة

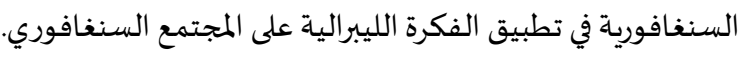

دراسة شارلين تانج (2008) بعنوان: Re)imagining the Muslim identity in Singapore هدفت الدراسة إلى بيان محاولة الحكومة في إعادة التشكيل عن شخصية المسلم في سنغافورة بعد الأزمة المتعلقة بالإرهابية التي قبضت في سنة 2002م. وقد خلصت الدراسة إلى أن الحكومة نجحت بقليل في مشروعيتها في إعادة التشكيل، وتخفيف المشاكل الإرهابية في سنغافورة. وسلكت الباحثة في الدراسة المنهج الوثائقي والتحليلي. وتتفق دراسة شارلين تانج، والدراسة الحالية في بيان

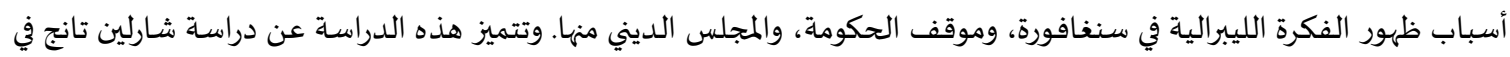
بيان سبب الفساد لهذه الفكرة الليبرالية من وجهة نظر الإسلام. مراسة نور الفديّة جوهري (2016) بعنوان: FEARING THE ENEMY WITHIN: A STUDY OF INTRA-MUSLIM يان PREJUDICE AMONG SINGAPOREAN MUSLIMS هدفت الدراسة إلى بيان أسباب الاختلاف، والنزاع بين المسلمين، وخصوصًا بين القوم الملاويين المسلمين في سنغافورة. ومن هذه

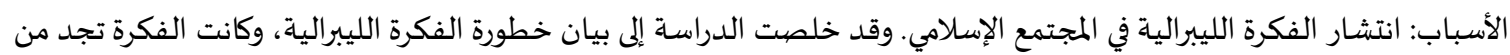
يدعم، ويتعارض بشكل واسع وبليغ من قبل كل من الحكومة، والمجلس الديني الإسلامي. وسلكت الباحثة في الدراسـة المنهج الوثائقي

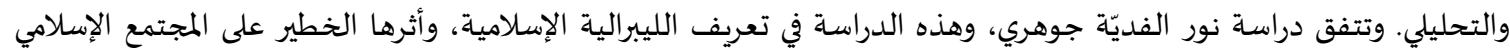

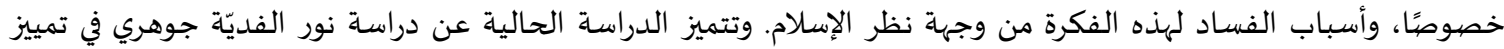

المقصود بالليبرالية الإسلامية من ناحية التيار، والفكرة العامة.

$$
\begin{aligned}
& \text { وقد اقتضت طبيعة الدراسة أن تقسم إلى مقدمة وثلاث مباحث وخاتمة على النحو الآتي : } \\
& \text { المبحث الأول: التعريف بالمصطلحات الواردة في البحث } \\
& \text { المطلب الأول: مفهوم الليبرالية } \\
& \text { المطلب الثاني: مفهوم الليبرالية الإسلامية عند المجتمع السنغافوري }
\end{aligned}
$$




$$
\begin{aligned}
& \text { المطلب الثالث: التعريف بسنغافورة } \\
& \text { المطلب الرابع: مفهوم دراسـة تطبيقية واقعية }
\end{aligned}
$$

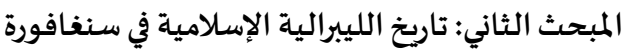

$$
\begin{aligned}
& \text { المطلب الأول: العوامل والأسباب المؤثرة في انتشار فكرة الليبرالية الإسلامية المباية } \\
& \text { المطلب الثاني: الآثار المترتبة من فكرة الليبرالية الإسلامية على المجتمع السنغافوري }
\end{aligned}
$$

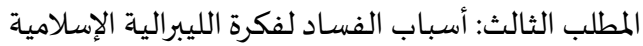

$$
\begin{aligned}
& \text { المطلب الرابع :وسائل الابتعاد عن فكرة الليبرالية الإسلامية }
\end{aligned}
$$

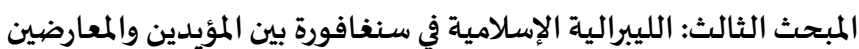

$$
\begin{aligned}
& \text { المطلب الأول: موقف الحكومة السنغافورية وآرائها عن فكرة الليبرالية الإسلامية بلية } \\
& \text { المطلب الثاني: موقف المجلس الديني الإسلامي وآرائه عن فكرة الليبرالية الإسلامية الماتهن }
\end{aligned}
$$

الخاتمة

\section{المبحث الأول: التعريف بالمصطلحات الواردة في البحث}

المطلب الأول: مفهوم الليبرالية

الليبرالية: مصطلح أجنبيّ معرَّب، مأخوذ من (Liberalism) في الإنجليزية، و(Liberalisme) في الفرنسية، وهي تعني: (التحررية)،

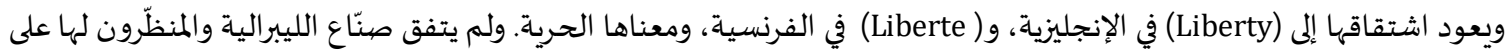
تعريف يحدد معناها بوضوح، لكنهم اتفقوا على وصفها بـالالحرية المطلقةشان.

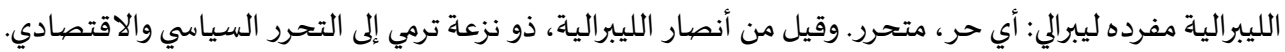

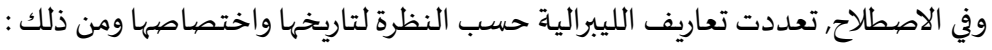

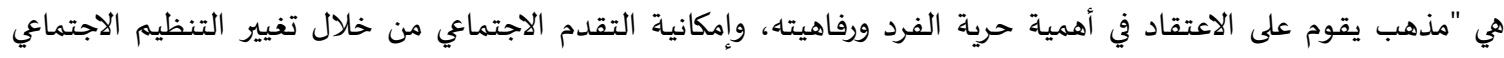

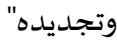

وقيل: "هي مذهب فكري يركز على الحرية الفردية ويرى وجوب احترام استقلال الأفراد، ويعتقد أن الوظيفة الأساسية للدولة هي

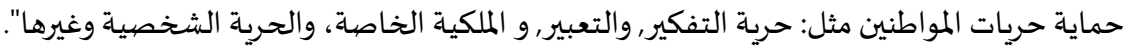

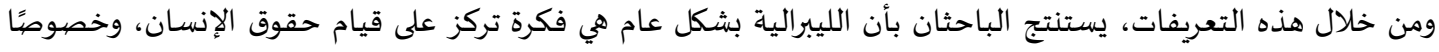

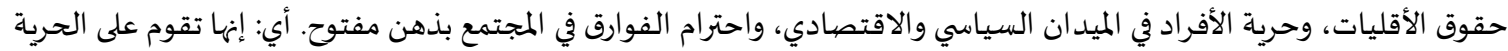

وقد قيل أن أصل الليبرالية هي من فكرة العلمانية أي: فصل الدين عن كل شيء. والعلمانية تعرف في أوروبا ب "ألّا يكون

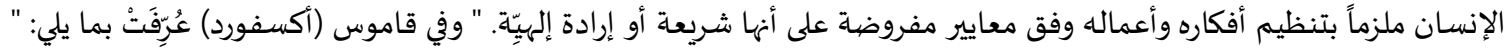
العلمانيَّة مفهوم يرى ضرورة أن تقوم الأخلاق والتعليم على أساس غير التهاير ديني."

المطلب الثاني: مفهوم الليبرالية الإسلامية عند المجتمع السنفافوري

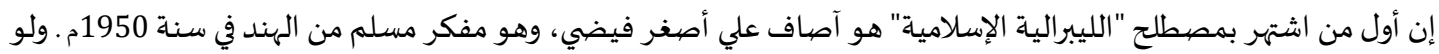
ننظر إلى التاريخ الإسلامي في القرن التاسع عشر الميلادي من الزعماء والكتاب مثل: جمال الدين الأفغاني, وسيد أحمد خان، ومحمد

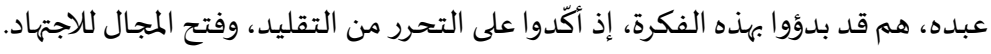

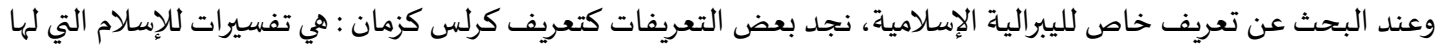

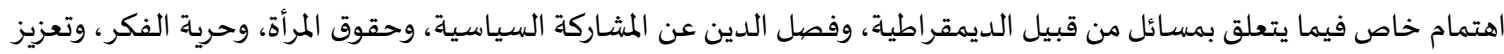

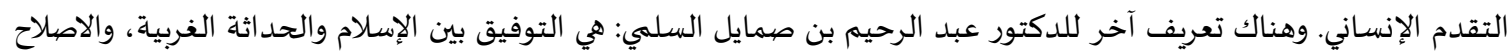

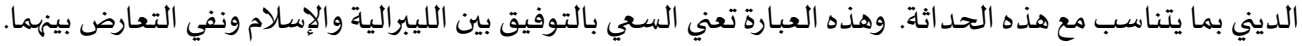

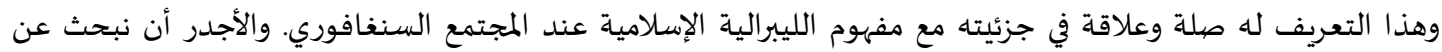
التعريف والمفهوم من خلال المواطن الذي يعيش في سنغافورة نفسه حتى نستوعب مرادها بالضبط. 
ومن خلال استقراء الباحثين الدراسات والبحوث عن البيئة الإسلامية في سنغافورة, نجد من أبرز التعريفات لليبرالية الإسلامية أنها عبارة عن فكرة وصورة الإسلام المعتدل حتى يكون الإسلام مناسبًا مع تطور الزمان. وقيل هي عبارة عن قدرة الفرد على احترام

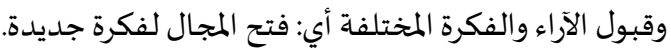

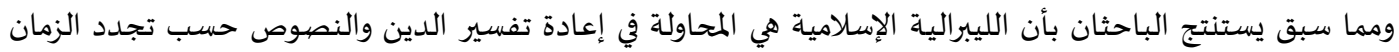

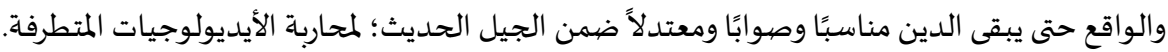

$$
\text { المطلب الثالث: التعريف بسنغافورة }
$$

سنغافورة: هي جمهورية تقع على جزيرة في جنوب شرقي آسيا، عند الطرف الجنوبي من شبه جزيرة ملايو، ويفصلها عن ماليزيا

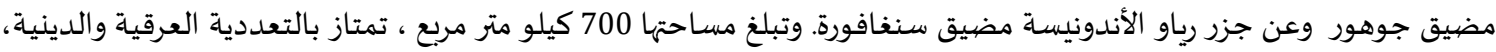
حيث يتألف المجتمع السنغافوري من 80\% من الصينيين، و 14\% من المالاي، و8\% من الهنود، و1\% من الأورآسيويين والأعراق

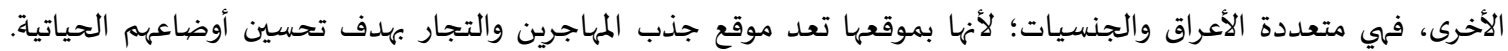
وسنغافورة بلد متعدد الديانات بسبب الخليط العرقي المتواجد فهها، وتقر الدولة في سنغافورة بحرية الاعتقاد، وفصل الدين عن الدين

الدولة.1 وخلاصية القول سنغافورة هي بلد صغير بوحدها، ولا عاصمة لها. أيضًا, هي بلد متعدد الأجناس والأديان والأقوام. وكلهم

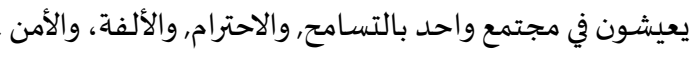

المطلب الر ابع: مفهوم دراسـة تطبيقية و اقعية كلمة "دراسة" تعني القراءة، وتحصيل العلوم والمعارف. وكلمة "تطبيقية" تعني الأمور التي تكون نتيجة تطبيق نظريات لمعرفة نتائجها. أما "واقعياة"، فهي الحقائق الناتجة عن الواقع. فمن هذه المعاني اللغوية, يستنتج الباحثان بأن مفهوم "دراسة تطبيقية واقعية" هي دراسة علمية تبين القضائ القضايا الراهنة والحقيقياة، والعملية مما لها علاقة وأثر على الحياة الفردية والاجتماعية في بلد معين.

المبحث الثاني: تاريخ الليبرالية الإسلامية في سنغافورة سنذكر في هذا المبحث أبرز العوامل والأسباب التي أدت إلى ظهور فكرة الليبرالية الإسلامية في سنغافورة، والآثار المترتبة على المجتمع والأفراد بسببها، وبيان فساد هذه الفكرة ووسائل الابتعاد عنها. المطلب الأول: العوامل و الأسباب المؤثرة في انتشارفكرة الليبرالية الإسلامية فكرة الليبرالية موجودة في المجتمع السنغافوري منذ زمن، لكن لم تظهر، وتنتشر بوضوح إلا في وقتنا الحاضرة، فقد توست توسعت واشتهرت حتى أصبحت من المشاكل المعروفة والخطيرة حول المجتمع وخصوصاً المسلمين. فبهذه الأسباب نلجأ إلى بحث جذور هذه

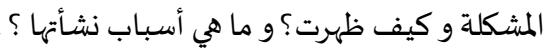
ومن خلال الملاحظة، واستقراء البحوث، والنصوص، والأخبار، يستنتج الباحثان الأسباب التي أدت إلى ظهور فكرة الليبرالية الإسلامية في سنغافورة.و هذه الأسباب هي: 1. السياق والواقع في البلد والمجتمع: سنغافورة بلد علماني، يتسم بتنوع الأديان والأقوام والأعراق فيها. وكل مواطن يعيش بالأمن فالأمن

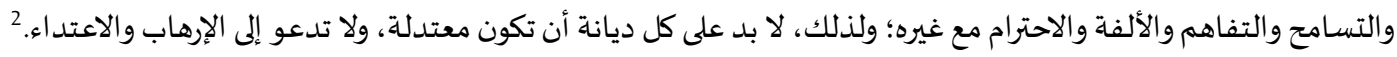
وقد صدر الإعلان في عام 2003م عن شأن "الانسجام الديني" من الهيئات الوطنية لكافة الجماعات الدينية الرئيسة في سنغافورة. وهذا الإعلان يخدم كأسـاس السنغافوريين جميعًا، وهو يعكس بصهورة منتظمة عن حالة الوئام الديني، وعن الخطوات التي يمكن أن نتخذها لتعميق علاقاتنا والتفاهم مع الطوائف الأخرى. 2. مشكلة الإرهاب: كانت سنغافورة معروفة بالحفاظ على نفسها خالية من الإرهاب، ومع ذلك كان التذكير دائم للمجتمع بأن يكون

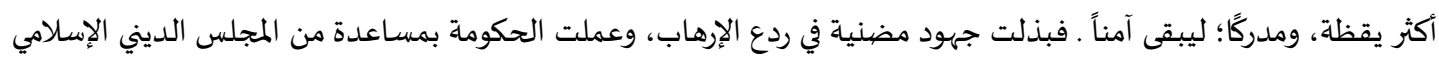
على مواجهة هذه المشكلة، ومن أبرزها إلقاء الندوات عن التسامح، والاحترام بين الأديان، وتفسير النصوص حسب الودهاء الواقع، 


$$
\text { ودعوة المحاضرين من الغرب لإلقاء الخطاب العام. }
$$

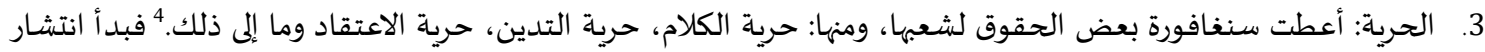

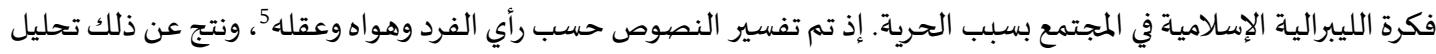

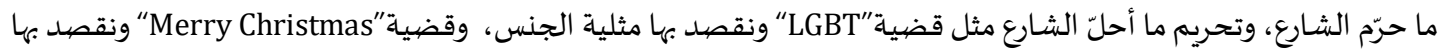
عيد الميلاد، وقضية الحجاب للمرأة، والتشكيك ببعض أحاديث النبي عليه الصلاة والسلام مثل: حديث الذبابة، وسن عائشـة رضي الله عنها حين تزوجت من النبي عليه الصلاة والسلام، وغيرها من الأمثلة.

4. قضية الاجتهاد: ومعنى ذلك القول بأن شريعة محمد عليه الصلاة والسلام تصلح وتتناسب مع زماناه، والأعراب السـابقين، فلا

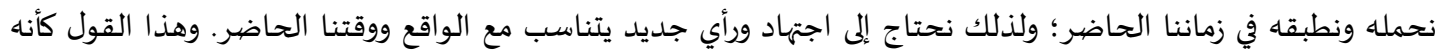

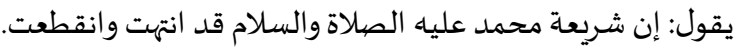
5. التعلم الديني في بلاد غير المسلمين: أحد الأسباب التي أدت إلى ظهور فكرة الليبرالية الإسلامية هي طلب العلم الشرعي والديني في بلاد غير المسلمين. حيث بدأوا عند رجوعهم لبلادهم بكتابة الرسائل، والبحوث، وإقامة التقارير، والتصيريحات الغريبة؛ وذلك

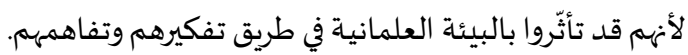
6. الدعم من الحكومة، والأديان الأخرى والشعوب العامة: وذلك لأنها في نهاية الأمر، فإن فكرة الليبرالية الإسلامية تؤدي إلى السلام والانسجام والوحدة في البلاد. فليس هناك أي سبب للحكومة أن تمنع من انتشار هذه الفكرة في المجتمع. فتجتهد الحكومة في

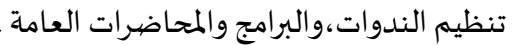

7. قضية الأقلية: يشكل الدين الإسلامي في سنغافورة بشكل عام, نسبة قليلة مقارنة بالأديان الأخرى. وتذكر الإحصائيات كما

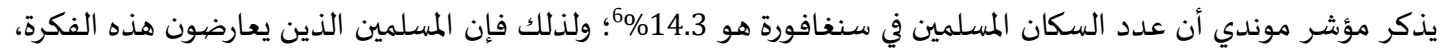
ليس لهم الحق في الاعتراض والاحتجاج سوى التعبير عن رأيهم أمام الناس. إضافة إلى ذلك، كانت فكرة الليبرالية الإسلامية عند ئد الحكومة، ليس لها أي مشكلة تتعلق بالإرهاب أو السياسـة، وهذا سمح بانتشار الفكرة في المجتمع؛ لأنها تتفق مع القوانين،

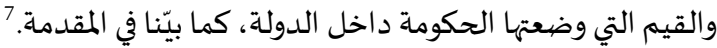
8. خارج السيطرة: لا شك أن هذه الفكرة قد انتشرت بقوة في المجتمع السنغافوري، فلا يستطيع أحد أن يوقفها من الانتشار في المجتمع. زيادة إلى التأييدات من الحكومة والمجتمع الآخر، فإيقاف هذه الفكرة أصبحت شباه مستحيلة في المجتمع

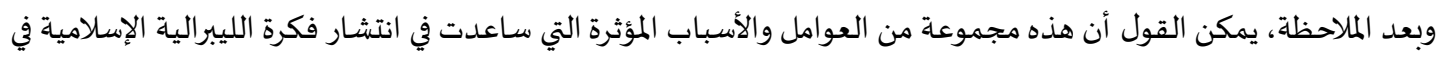

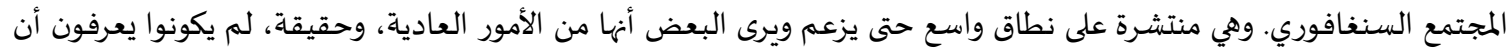

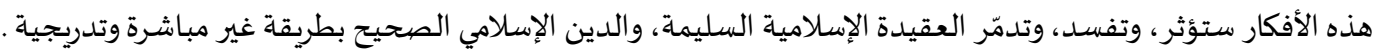
المطلب الثاني: الآثار المترتبة على فكرة الليبرالية الإسلامية في المجتمع السنغافوري في هذا المطلب، سنقسم الآثار المترتبة على هذه الفكرة في المجتمع السنغافوري إلى قسمين هما: الأول: الآثار السلبية على

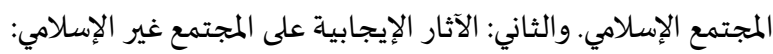

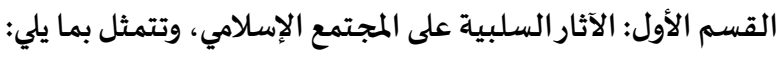
1. الفساد الديني: أي: الفساد الذي يكون في فهم التشريعات الصحيحة التي شرعها الله عزّ وجلّ، وأنزلها على النبي محمد - صلى الإسلى

2. الفساد الخلقي: أي: الفساد الذي يكون في أخلاق وسلوك المسلم حيث يفعل أي شيء مما يفسده، ويؤذيه مثل: القضية التي

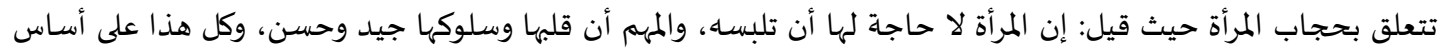

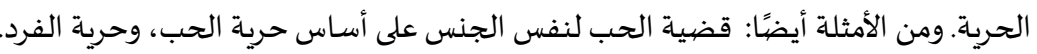
3. الفساد الاعتقادي: أي: الفساد الذي يكون في إيمان وعقيدة المسلم، وكما بيّنا أن من أحد الأقوال المشهورة لهذه الفكرة أن

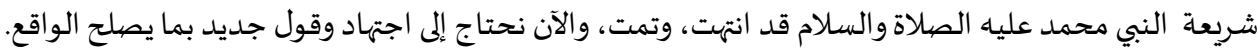

${ }^{4}$ https://sso.agc.gov.sg/Act/CONS1963?ProvIds=P1IV-\#pr13-

5 http://www.nzasia.org.nz/downloads/NZJAS-Dec05/7_2_3.pdf

${ }^{6} \mathrm{https}: / /$ www.indexmundi.com/singapore/demographics_profile.html

${ }^{7}$ https://www.ircc.sg/ABOUT\%20IRCC/Vision\%20and\%20Mission 


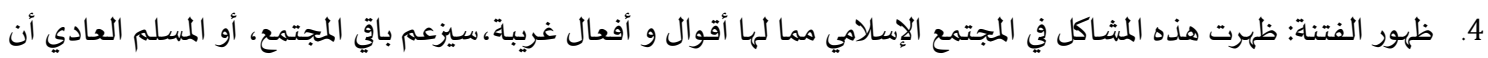

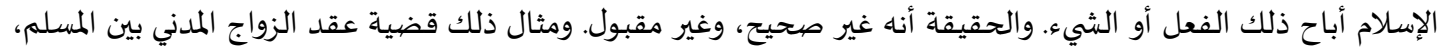

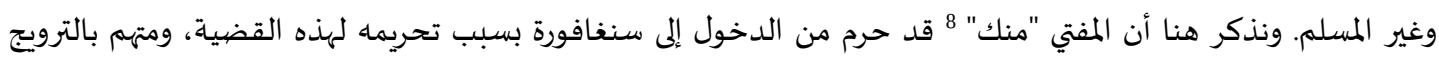

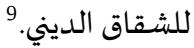

القسم الثاني: الآثار الإيجابية على المجتمع غير الإسلامي.

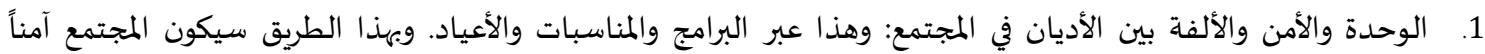

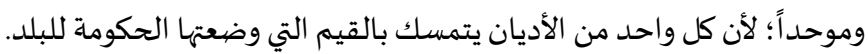

2. السمعة الطيبة والصورة المشرقة للبلد: لما كانت سنغافورة ستبقى على سلام وأمان دون أي اعتداء واحتدام، لا شك أنها لأنا ستظهر بصورة مشرقة أمام العالم. 3. العائد الاقتصادي: من الطرق التي سلكتها سنغافورة في الحصول على المال الترويج لبلدها عبر التجار والزائرين من الدول

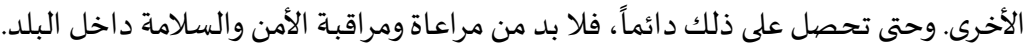

المطلب الثالث: أسباب فساد فكرة الليبرالية الإسلامية

لا شك أن هناك أسباب كثيرة في عدم قبول فكرة الليبرالية الإسلامية؛ لما فهيها من الفساد الكبير على عقيدة المسلم، وأخلاقه،

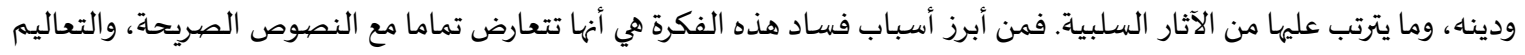

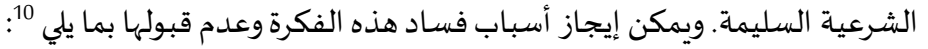

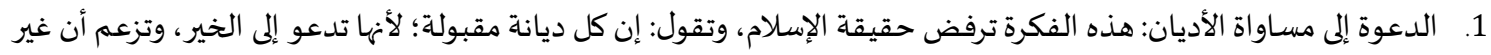

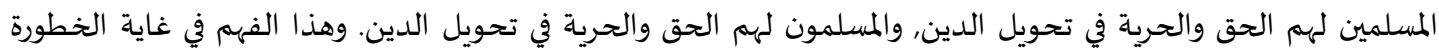

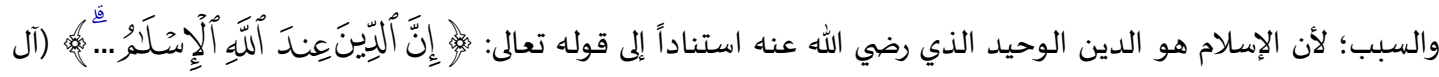

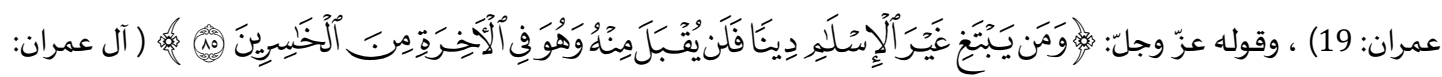

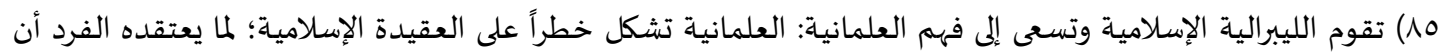

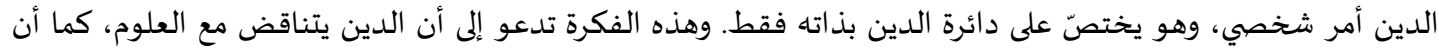

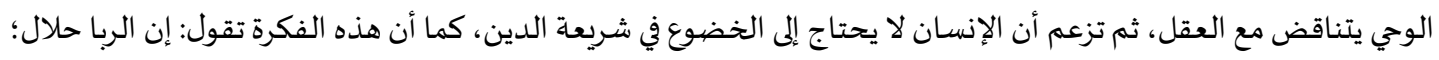
لأنه يساعد في مساهمة الاقتصاد داخل البلد، وأن شرب الخمر يعتبر من حقوق الأفراد التي لا يستطيع تشويشها.

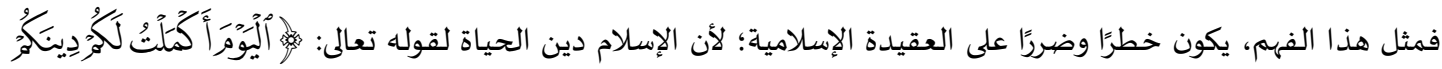

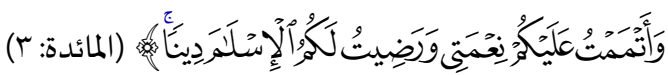

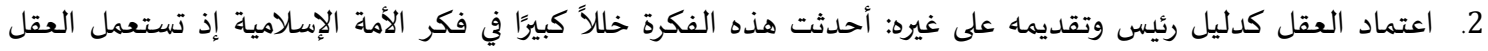

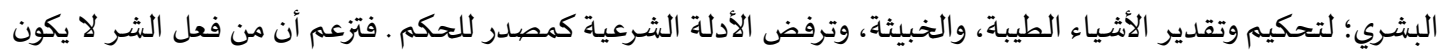

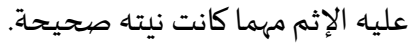
ومثل هذا الفهم، لا شك أنه خطأُ لأن القاعدة الفقهية تقول: إن الغاية لا تبرّّر الوسيلة. أي ولو كانت النية صحيحة، لا بد أن أن تكون وسيلتها وكيفيتها صحيحة مضبوطة بالدليل الشرعي.

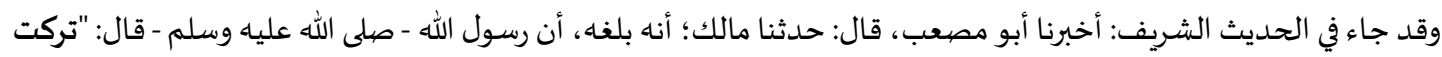

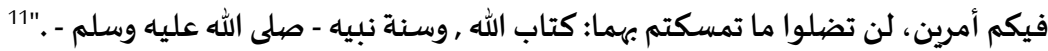

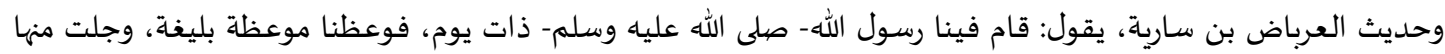

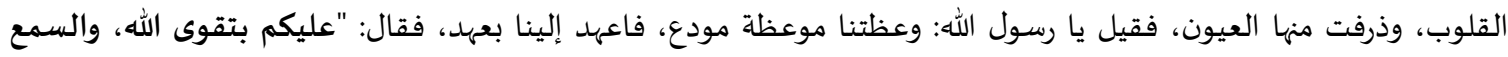

8 هو اسماعيل بن موسى منك المفتي الأكبر في زيمبابوي . ${ }^{9}$ https://www.aljazeera.com/news/2017/10/singapore-bans-mufti-menk-entering-country-171031064359778.html ${ }^{10} \mathrm{http}: / /$ emasjid.jais.gov.my/uploads/uploads/21.09.2018\%20(RUMI)\%20FAHAMAN\%20LIBERAL\%20PEROSAK\%20AKIDA H.pdf 
والطاعة، وإن عبدًا حبشيًًا، وسترون من بعدي اختلافًا شـديدًا، فعليكم بسـني، وسـنة الخلفاء الراشدين المهديين، عضهوا عليها

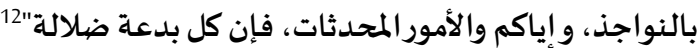

ونكتفي بذكر هذه الأدلة والأسباب التي تدل على فساد فكرة الليبرالية الإسلامية. ومن هنا يرى الباحثان أن الليبرالية الإسلامية هي مصطلح غير صحيح أصلاً؛ لأنهما كلمتان نقيضتان. الليبرالية من أحد معانها كما ذكرنا الحرية. والإسلام بمعنى الانقياد

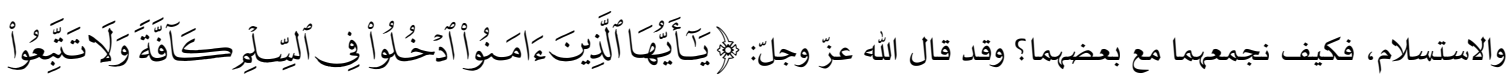

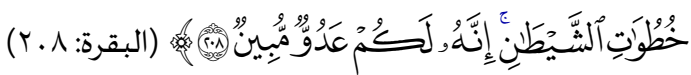

المطلب الر ابع: وسائل الابتعاد عن فكرة الليبرالية الإسلامية

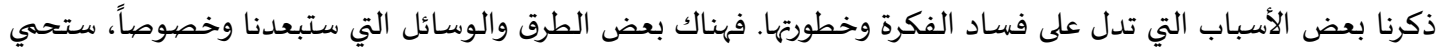

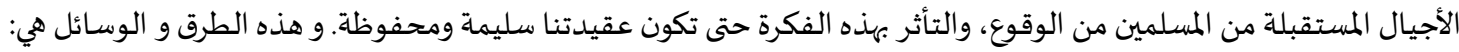
1. التمسك بكتاب الله، وسنة رسوله عليه الصلاة والسلام، وفهم السلف الصالح في أمونه المور العقيدة، والشريعة والأخلاق؛ لأن فكرة الليبرالية الإسلامية عند أصحابها، تزعم أهها تتمسك بالقرآن والسنة، لكن ليس على فهم صحيح، بل على فهيه فههم وهواهم، وبما

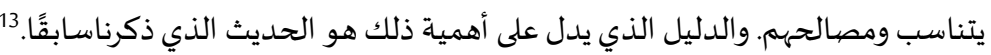
2. التربية الإسلامية الصحيحة لأولادنا منذ الصغر. فدور الأبوين مهم في حياة الأولاد، وله أثر كبير. كما قال الله تعالى في قصاية

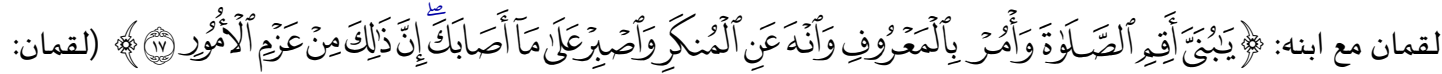

وكما جاء عن عمرو بن شعيب، عن أبيه، عن جده، قال: قال رسول الله - صلى الله عليه وسلم - : "مروا أولادكم بالصهلاة وهم

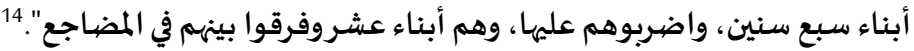

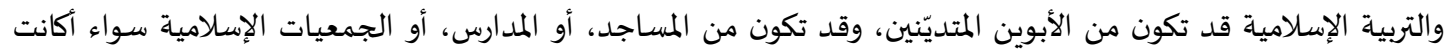
دوامها كامل أم جزئي؛ لأن بعض الأسر في سنغافورة ليست متديّنة بشكل كبير, فتستطيع أن ترسل أولادها إلى هذه الأماكن المذكورة.

3. المداومة بطلب العلم الصحيح مع المعلّم الصحيح, المعتبر والمعروف. فعلينا أن نستمر بطلب العلوم الشرعية طول الحياة، وأن نعرف ممن نطلب العلم. فالمعلّم الصحيح ينشر العلم الصحيح، وكذلك العكس. كما في صحيح مسلم عن محمد بن سيرين، قال :"إن هذا العلم دين، فانظروا عمن تأخذذون دينكم" وقد صدر القانون الإلزامي من المجلس الديني الإسلامي، وجمعية العلماء، ومدرسي الدين الإسلامي بسنغافورة الذي يشير إلى

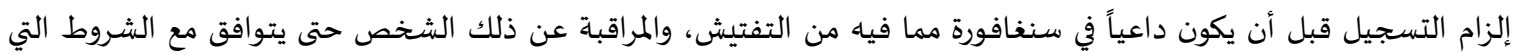

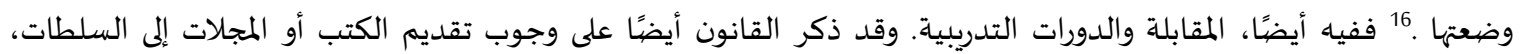
والحصول على القبول منها قبل نشرها حتى يضمن عدم وجود فكرة الإرهاب أو الاعتداء، وما يشبه ذلك في مضيمونها. 17 وكان الهدف في هذه الأوامر هو التأكيد للمجتمع على أن المعلمين الذين يقدمون الإرشاد والتعليم الديني مؤهلون ومدربون هذا الأمر لله إيجابيات وسلبيات. فمن الإيجابيات أن العلوم الدينية المنتشرة في سنغافورة صارت سليمة من فكرة الإرهاب، والمجتمع يكون مطمئناً. ومن السلبيات التي لا تظهر بشكل مباشر الدورات التدريبية سواء أكانت للأساتذة المحليين أم للمجتمعح, ففيها

$$
\begin{aligned}
& 12 \text { القزويني، ابن ماجه - سنن ابن ماجها، ج1، ص15,باب اتباع سنة الخلفاء الراشدين المهديين، ح42. }
\end{aligned}
$$

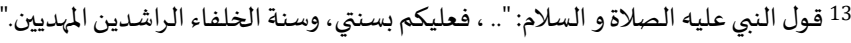

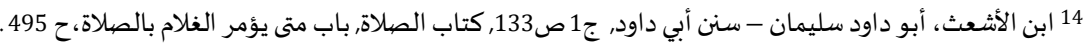

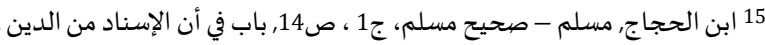

${ }^{16}$ https://www.channelnewsasia.com/news/singapore/asatizah-recognition-scheme-to-become-compulsory-from-january20-7799112

${ }^{17}$ https://www.straitstimes.com/singapore/islamic-schools-have-to-get-muis-approval-before-introducing-new-religioustextbooks

${ }^{18} \mathrm{https}$ //www.todayonline.com/singapore/malaymuslim-leaders-support-compulsory-registration-islamic-religiousteachers-spore 
غزو الفكر ، والدعوة إلى فكرة الليبرالية الإسلامية. فينادوا المحاضرين الغربيين؛ ليعلّموا ويدرّبوا الإسلام حسب الواقع، أو المخفي تحت اسم "الوسطية" لكن أفكاره غريبة كتشكيك ببعض الأحاديث الشريفة لتحقيق المصالح الخاصة،، وما إلى ذلك.

وهذا الأمر قد يرى بعض الناس أنه شيء إيجابي؛ لأنه يدعو إلى الأمن، والتسامح مع المجتمع، والأقوام، ويتناسب مع واقع

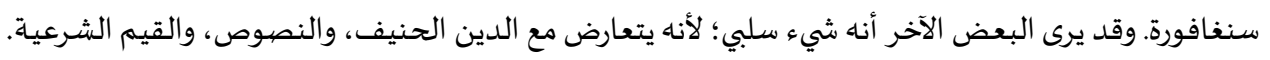

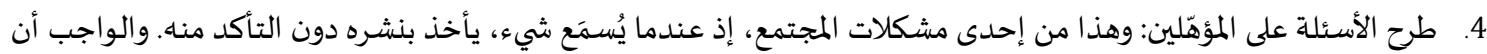

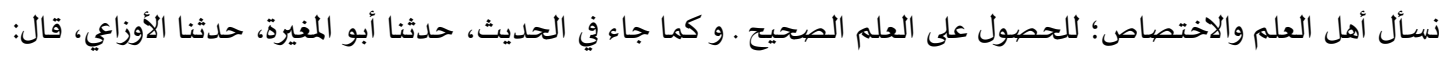

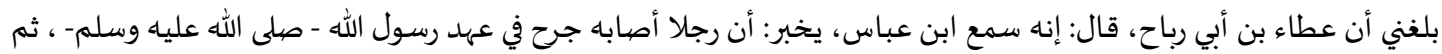

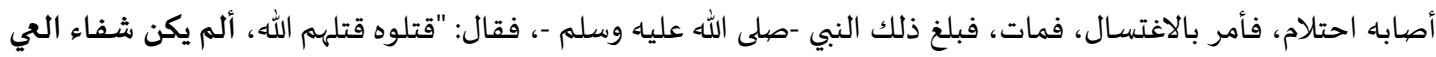
السؤال". 19

5. عدم تتبع الأهواء في مسألة ما، وعلينا الرجوع إلى كتاب الله، وسنة رسوله عليه الصلاة والسلام، وأقوال السلف الصالح؛ لأن كل

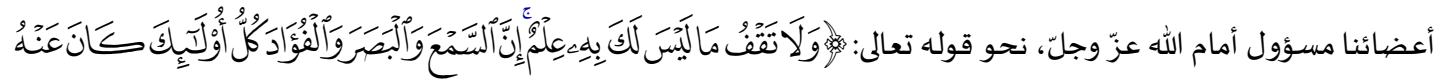

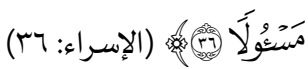

6. لا شك أن هناك طرق ووسائل كثيرة للابتعاد عن هذه الفكرة الخطيرة، لكن هذا مما يستطاع معرفته واستنتاجه من خلال الاستقراء، والخبرة كالمواطن الذي يعيش في تلك البلاد .

\section{المبحث الثالث: الليبرالية الإسلامية بين المؤيدين و المعارضين}

المطلب الأول: موقف وآراء الحكومة السنغافورية من فكرة الليبرالية الإسلامية

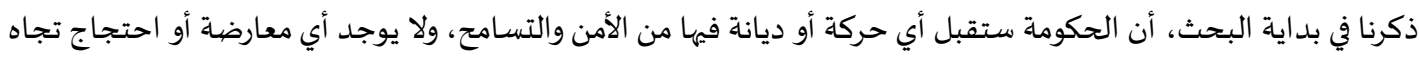

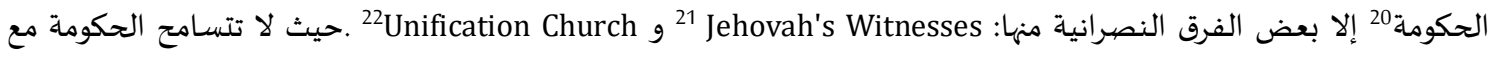

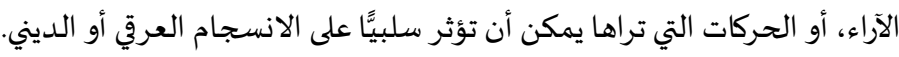

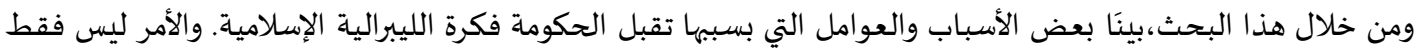
تقبل الحكومة هذه الفكرة، بل تساعد في نشرها من كل الجوانب؛ لما فيها من الأمن والتسامح. والدليل الذي يدل على مشـاركة الحكومة في انتشار هذه الفكرة هو إنشاء الندوات والمؤتمرات 23 ونشر الكتب والرسالات. وخلاصة الفهرة الأمر أن الحكومة من من المؤيدين

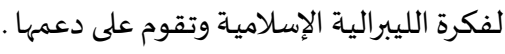

المطلب الثاني: موقف و آراء المجلس الديني الإسلامي عن فكرة الليبرالية الإسلامية في هذا المطلب سنذكر موقف المجلس الديني الإسلامي، وموقف جمعية العلماء ومدرسي الدين الإسلامي على أهها تعد من

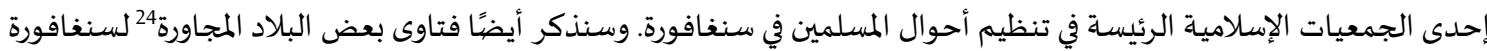
، وبيان ذلك: أولاً: موقف المجلس الديني الإسلامي: من خلال الاستقراء، يرى الباحثان أن موقفه واضح وصريح، وإنما يقف المجلس الديني

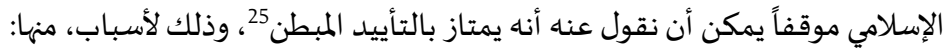
1. ق قيل: إن بعض العمال والموظفين داخل المجلس يميلون إلى هذه الفكرة. وهذا الأمر قد ظهر جليًا في السياسات والقوانين داخل المجلس. مثل: استقبال زيارة العلماء الغربيين الليبراليين، وتوفير المنح الدراسية لمرحلة الدراسات العليا في الجامعات المعروفة

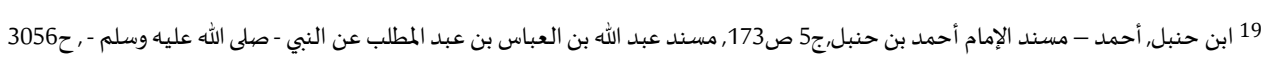
http://www.iro.sg/و https://www.ircc.sg/ 20

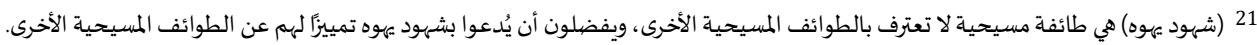

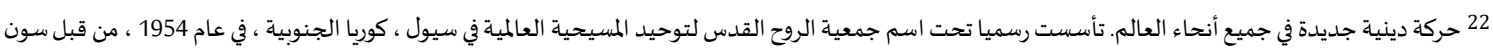
${ }^{23} \mathrm{http} / / /$ iro.sg/our-events/

ميونغ مون. 25 https://www.facebook.com/AlmakhazinSG/posts/a-comment-by-syed-danial-to-mufti-fatris-bakarams-posting-on-thehijab-issuebism/420736384723739/ 
بالإيديولوجيات الليبرالية.

2. هذا المجلس تحت رعاية الحكومة27، فلا يستطيع أن يخالف أمر الحكومة، وعليها تنفيذ كل ما تطلبه الحكومة. وقد تشاركا مع

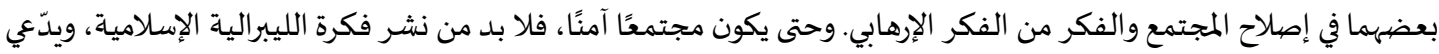

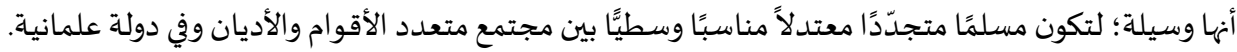

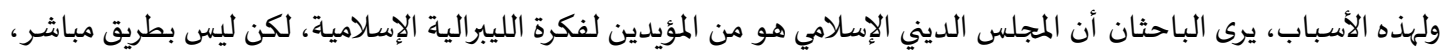
ويختفي تحت مصطلح "الوسطية " لكن ليس على مفهوم صحيح.

ثانياً: موقف جمعية العلماء ومدرسي الدين الإسلامي: ذكرنا أن هذه الجمعية هي إحدى الجمعيات الإسلامية الرئيسة التي تنظّم

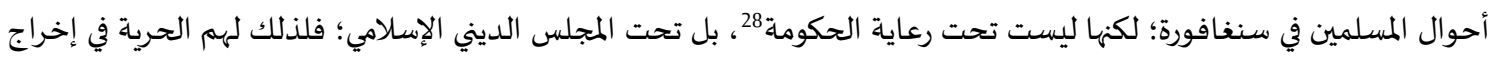
الآراء والأقوال. فعندهم أقوال متعلقة بقضية الميليبرالية بشكل عام وما يترتب عليها من أفكار مثل: قضية "LGBT"

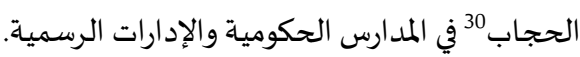
فقولهم عن الليبرالية هو: "أن الليبرالية غالبا تنتسب إلى التقدم والإنمائية والحداثلة المداثة. وكون الفرد ليس ليبراليا يعتبر متخلّف

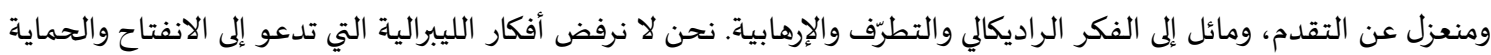
على حقوق الإنسان. بل إنما نرفض الليبرالية من الناحية التي تسبق مصلحة الفرد على مصلحة العامة. أيضًا، نرفض الفئا أفكارها التي تميل إلى التساهل وHedonism

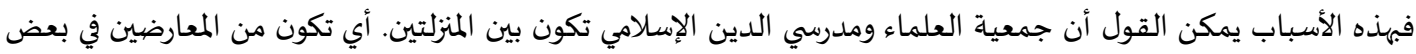

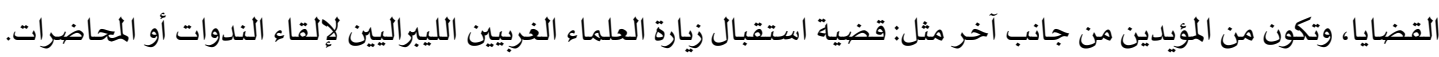
كما قلنا: إن هذه الجمعية تحت رعاية المجلس الديني الإسلامي، ومع أن هذا المجلس تحونس تحت رعائل رعاية الحكومة، فكل التعاليم

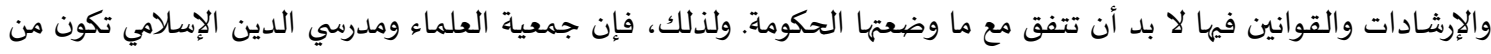

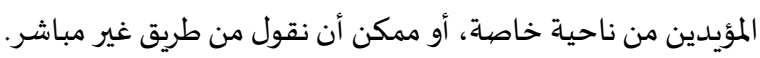

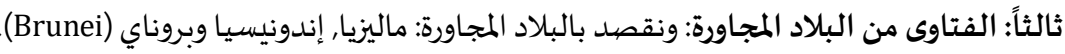

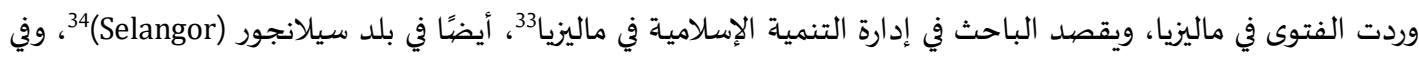

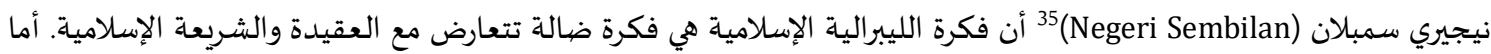

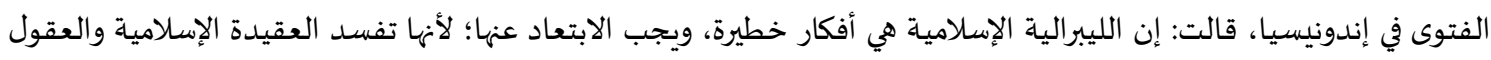

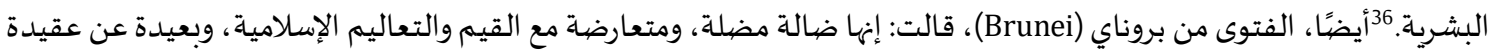

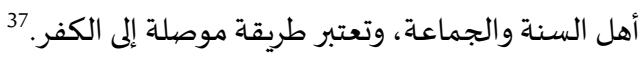
ونلاحظ من خلال ما سبق أن هذه الفتاوى تدل على أن هذه البلاد ترفض رفضيًا تامَّا هذه الفكرة، وهي من المعارضين لها.

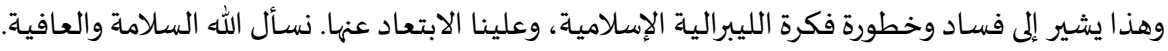

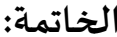

الحمد لله الذي بنعمته تتم الصالحات، والحمد لله أولاً وآخرًا وظاهرًا وباطنًا، حمدًا كثيرًا طيبًا مباركًا كما ينبغي لجلال وجهها

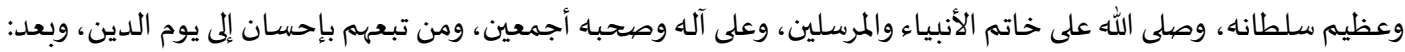

${ }^{26}$ https://rilek1corner.com/2014/03/08/the-reading-group-muis-and-liberal-islam/

${ }^{27}$ https://www.muis.gov.sg/About-MUIS/History

${ }^{28} \mathrm{https} / / /$ www.pergas.org.sg/about-us.html

${ }^{29} \mathrm{http}: / /$ www.pergas.org.sg/media/MediaStatement/English-Pemansuhan-Seksyen377A.pdf ,

http://www.pergas.org.sg/media/MediaStatement/Media-Statement-Pergas-response-to-HPB-FAQ-on-Sexuality.pdf ${ }^{30} \mathrm{http} / / /$ www.pergas.org.sg/media/MediaStatement/Media-Statement-Tudung-Issue-8-November.pdf

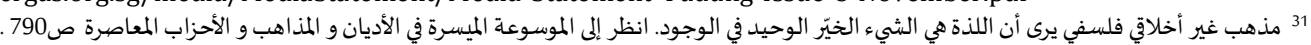

صoderation in Islam : in the context of Muslim community in Singapore ${ }^{32}$

${ }^{33} \mathrm{http}: / /$ www.islam.gov.my/images/ePenerbitan/KOMPILASI_MUZAKARAH_MKI_2016.pdf

$34 \mathrm{http} / / /$ www.muftiselangor.gov.my/2016-06-27-08-57-00/fatwa/fatwa-terkini/362-fatwa-pemikiran-liberalisme-danpluralisme-agama

35 http://www.muftins.gov.my/index.php/arkib2/himpunan-fatwa/171-keputusan-mesyuarat-fatwa/713-aliran-pemikiranliberal-hukum-dan-implikasinya-kepada-islam

${ }^{36} \mathrm{http}: / /$ mui.or.id/wp-content/uploads/2017/02/12b.-Penjelasan-Tentang-Fatwa-Pluralisme-Liberalisme-dan-Se.pdf ${ }^{37} \mathrm{http}$ // $/$ www.kheu.gov.bn/lists/khutbah/allitems.aspx?Paged=TRUE\&PagedPrev=TRUE\&p_ID=15\&PageFirstRow=841\&\& View=\%7B50C61C94-532E-45DB-82E1-C3FB48D801DB\%7D \#InplviewHash50c61c94-532e-45db-82e1-c3fb48d801db= 
يسّر الله لنا بفضله وكرمه ونعمه التي لا تحصى بإتمام كتابة هذا البحث الموجز والمفيد إن شـاء الله. ولا نزعم أن هذا البحث قد بلغ الكمال، أو خلا من الأخطاء والنقصان، ولكن الذي نؤكد عليه أنه قد بذلنا فياه الجهد، فإن أصبنا فذلك توفيق من الله، وما كان فياه من خطأ ونقصان فمن أنفسنا.

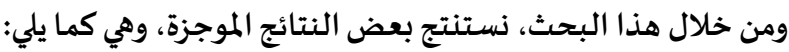
الليبرالية الإسلامية لها مفهوم خاص في سنغافورة، وهو المحاولة في إعادة تفسير الدين والنصوص حسب تجدد الزمان والواقع

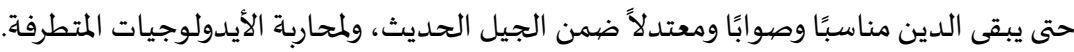
الليبرالية الإسلامية هي فكرة فاسدة عند عرضها على العقيدة الإسلامية الصحيحة والشريعة والأخلاق مهما كان ظاهرها وهدفها

موقف الحكومة من فكرة وتعليم الليبرالية الإسلامية مقبولة لديها، وغير مجابة لدى المجلس الديني الإسلامي. أما موقف جمعية العلماء ومدرسي الدين الإسلامي، فيكون بين المنزلتين وعلى حسب الأحوال. الليبرالية الإسلامية لها آثار إيجابية وسلبية على الحكومة السنغافورية وسياستها، وعلى المجتمع السنغافوري من المسلمين

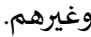
الليبرالية الإسلامية لا تزال منتشرة في المجتمع السنغافوري، وكأنه من المستحيل إيقافها والابتعاد عنها. ويوصي البحث في ضيوء هذه النتائج ب : التمسك والالتزام بكتاب الله وسنة رسوله على فهم السلف الصالح الصحيح. التربية الإسلامية الصحيحة للأسرة والأولاد منذ صغرهم. كثرة القراءة، وطلب العلم، وحضور المجالس العلمية الصحيحة.

ونرجو أن القارئ يستفيد من هذا البحث البسيط، وعنده صورة عن الأحوال في سنغافورة وخصيوصًا الأمور المتعلقة بالدين

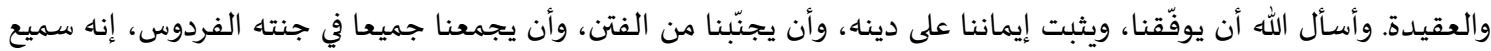

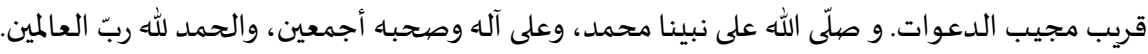

1. ابن الأشعث، أبو داود سليمان، سنن أبي داود. المكتبة العصرية. صيدا أبيروت بيروت. 2.

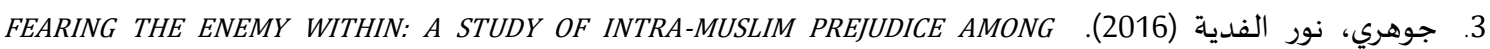
SINGAPOREAN MUSLIMS 4. ابن الحجاج، مسلم. صحيح مسله. دار إحياء التراث العربي- بيروت.

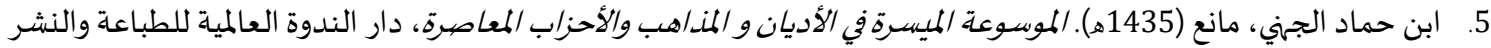
والتوزيع -الرياض، السعودية العربية. ط3.

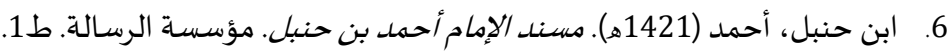
7. الخلف، أحمد بن عبد العزيز بن خلف (1437ه). الوجيز في المنداهب الفكرية المعاصرة عرض ونقل. دار الإمام مسلم, المدينة المنورة-السعودية العربية. ط1. 8. السلمي، عبد الرحيم بن صمايل (1430ه). حقيقة الليبرالية وموقف الإسلام منها. مركز التأصيل للدراسات و البحوث.جدةالسعودية العبربية. طب.

(Re)imagining the Muslim identity in Singapore (2008)

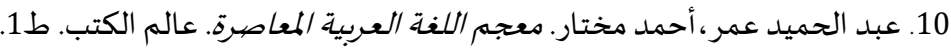
11. فرجاس (1) Moderation in Islam : in the context of Muslim community in Singapore(PERGAS) 12. القزويني، ابن ماجه أبو عبد الله محمد بن يزيد. سنن /بن ماجهاء دار إحياء الكتب العربية. 13. الكيلاني، آمال (2010). ترجمة من موسوعة ويكبيديا. القراءة للجميع. الهيئة العامة للكتاب. 
14. محمد صقر، شحانة. الإسالام والليبرالية نقيضان لا يجتمعان. دار الخلفاء الراشدين.

15. نور الدين أحمد علي. 1SLAM AND STATE: A STUDY OF THE LIBERAL ISLAMIC NETWORK IN INDONESIA, 1999-2004 همدين

16. /https://www.urbandictionary.com

17. /https://www.nzasia.org.nz

18. /https://www.an-naseehah.blogspot.com

19. https://sso.agc.gov.sg

20. http://iro.sg

21. https://www.ndtv.com/

22. http://www.nzasia.org.nz

23. https://www.indexmundi.com

24. https://www.ircc.sg/

25. http://emasjid.jais.gov.my /

26. https://www.channelnewsasia.com

27. https://www.straitstimes.com

28. https://www.todayonline.com

29. https://www.facebook.com/AlmakhazinSG//

30. https://rilek1corner.com

31. https://www.muis.gov.sg/

32. https://www.pergas.org.sgl

33. http://www.islam.gov.my/

34. http://www.muftiselangor.gov.my/

35. http://www.muftins.gov.my/

36. http://mui.or.id/

37. http://www.kheu.gov.bn/

38. https://www.aljazeera.com/news/ 


\title{
المجلة الدولية للدراسـات الإسلامية المتخصصية
}

International Journal of Specialized Islamic Studies (SIS)

Journal Homepage: https://www.refaad.com/views/SIS/Home.aspx ISSN: 2617-6246(Online) 2617-6238(Print)

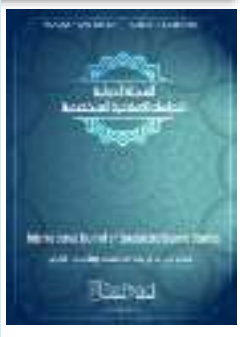

\section{Islamic Liberal in Singapore: An applied and Contemporary Studies}

\author{
Ra'ed Said Ahmad Bani Abed Alrahman \\ Assistant professor in Creed, Faculty of Al-Sharee'a and Islamic Studies, Yarmouk University, Jordan \\ raed.said@yu.edu.jo
}

\section{Mohammad Hafed Aldin Bin Khamees}

Researcher, Yarmouk University, Jordan

DOI: https://doi.org/10.31559/SIS2020.5.2.2

Abstract: The study aimed to demonstrate the concept of Islamic liberalism in the Singaporean society and to reveal its truth and the reasons that led to its emergence and its effects in society, as well as the reasons of corruption for this idea and mentioned the position of the government and the religious councils. Through a comparative documentary approach and an analytical approach.

The study concluded that Islamic liberalism has a special concept in Singapore, which is corrupt when it is presented to Islamic and Islamic belief and ethics, but accepted by the Singaporean government and is still widespread in society.

The most important recommendation of the study is to adhere to the book of Allah and his Messenger, peace be upon him where there is no way to focus only on the religious education and correct education.

Keywords: sects; intellectual; contemporary; Islamic liberalism; Singapore; Freedom; Islamic Religious Council; human rights; secularism.

\section{References:}

1. 'bd Alhmyd 'mr,Ahmd Mkhtar. M'jm Allghh Al'rbyh Alm'asrh. 'alm Alktb. T1.

2. Abn Ans, Malk. (412h). Mwta Alemam Malk. M'sst Alrsalh.

3. Abn Alash'th, Abw Dawd Slyman, Snn Aby Dawd. Almktbh Al'sryh. Syda- Byrwt.

4. Frjas (Pergas) Moderation in Islam: In the Context of Muslim Community in Singapore.

5. Abn Alhjaj, Mslm. Shyh Mslm. Dar Ehya' Altrath Al'rby- Byrwt.

6. Abn Hmad Aljhny, Man' (1435h). Almwsw'h Almysrh Fy Aladyan W Almdahb Walahzab Alm'asrh, Dar Alndwh Al'almyh Lltba'h Walnshr Waltwzy'-Alryad, Als'wdyh Al'rbyh. T3.

7. Abn Hnbl, Ahmd (1421h). Msnd Alemam Ahmd Bn Hnbl. M'sst Alrsalh. T1.

8. Jwhry, Nwr Alfdyh (2016). Fearing The Enemy Within: A Study of Intra-Muslim Prejudice Among Singaporean Muslims

9. Alkhlf, Ahmd Bn 'bd Al'zyz Bn Khlf (1437h). Alwjyz Fy Almdahb Alfkryh Alm'asrh 'Erd Wnqd. Dar Alemam Mslm, Almdynh Almnwrh-Als'wdyh Al'rbyh. T1.

10. Alkylany, Amal (2010). Trjmh Mn Mwsw'h Wykbydya. Alqra'h Lljmy'. Alhy'h Al'amh Llktab.

11. Mhmd Sqr, Shhanh. Aleslam Wallybralyh Nqydan La Yjtm'an. Dar Alkhlfa' Alrashdyn. 
12. Nwr Aldyn Ahmd 'ly. Islam And State: A Study of the Liberal Islamic Network in Indonesia, 1999-2004.

13. Alqzwyny, Abn Majh Abw 'bd Allh Mhmd Bn Yzyd. Snn Abn Majh. Dar Ehya' Alktb Al'rbyh.

14. Sharlyn, Tanj (2008) (Re)Imagining the Muslim Identity in Singapore.

15. Alslmy, 'bd Alrhym Bn Smayl (1430h). Hqyqh Allybralyh Wmwqf Aleslam Mnha. Mrkz Altasyl Lldrasat W Albhwth.Jdh-Als'wdyh Al'rbyh. T1. 